\title{
Simple and efficient method for carbon nanotube attachment to scanning probes and other substrates
}

\author{
A. Hall \\ Department Physics and Astronomy, University of North Carolina at Chapel Hill, \\ Chapel Hill, North Carolina 27599 \\ W. G. Matthews \\ Cystic Fibrosis Center, University of North Carolina at Chapel Hill, Chapel Hill, North Carolina 27599 \\ R. Superfine, M. R. Falvo, and S. Washburn ${ }^{a)}$ \\ Department of Physics and Astronomy, University of North Carolina at Chapel Hill, \\ Chapel Hill, North Carolina 27599
}

(Received 12 June 2002; accepted 17 February 2003)

\begin{abstract}
We present a fast, high yield, low cost method for the production of scanning probes with aligned carbon nanotubes protruding from the ends. The procedure is described and images of undercut films are used to demonstrate the improved probe quality for topography measurements. A magnetophoretic model of the attachment and alignment processes is discussed. (C) 2003 American Institute of Physics. [DOI: 10.1063/1.1567049]
\end{abstract}

There have been several attempts to use carbon nanotubes (CNT) as scanning probe sensors. Their high aspect ratio and mechanical resilience ${ }^{1,2}$ make them ideal candidates for force measurements, and their ideal electrical properties $^{3}$ imply utility in scanning potentiometry. Previous attempts have included chemical vapor deposition growth of a CNT directly onto commercial atomic force microscope (AFM) tips made of $\mathrm{Si}$ or one of its derivatives, ${ }^{4}$ and the mechanical attachment of a CNT onto an AFM tip. ${ }^{5-8}$ Finding these methods somewhat arduous, we have developed a method based on the liquid deposition of a CNT onto AFM tips. Recognizing the anisotropic tendency of a CNT to align along magnetic field lines, ${ }^{9,10}$ we have used magnetic fields to align the CNT along the AFM tip axis. In addition, this method may be amenable to attaching the CNT to almost any conductive material.

An apparatus was assembled in order to introduce a magnetic field onto a single AFM probe and a nanotube suspension, as shown in Fig. 1. Here, a custom-made beaker is positioned above a solenoid ( 340 turns) containing a high permeability, type I, low carbon magnetic iron core (Scientific Alloys, Westerly, RI). The beaker was designed in such a way as to minimize the distance between the AFM tip and the solenoid core. This was accomplished by attaching a glass cover slip with a shelf, which serves as a platform for the probe chip, to a glass cylinder ( $\sim 20 \mathrm{~mm}$ diameter). This positioned the AFM tip about $400 \mu \mathrm{m}$ from the solenoid tip. Into the beaker was introduced $5 \mathrm{~mL}$ of carbon arc-synthesized $^{11}$ multiwalled CNTs suspended in dichloromethane. Prior to this introduction, the suspension was sonicated for 10-15 min to ensure maximum homogeneity. An AFM probe was sputter coated with $60 \mathrm{~nm}$ of gold and introduced, tip-side down, onto the submerged platform. The probe tip was visually aligned above the pole piece. An alternating current of $7 \mathrm{~A}$ at $60 \mathrm{~Hz}$ was then applied to the

${ }^{a)}$ Electronic mail: sean@physics.unc.edu solenoid, resulting in a measured magnetic field amplitude $B_{0} \approx 0.1 \mathrm{~T}$. With this apparatus, the anisotropic properties of the CNT cause the tubes that come into contact with the probe tip to be preferentially oriented parallel to the tip direction $^{9}$ and hence protruding down from the end.

Using this method, with the solenoid energized for 1 min, we obtain potentially useful CNT-tipped probes (i.e., probes with a nanotube protruding past the end of the AFM tip) in about $50 \%$ of the trials. Examples of processed tips can be seen in Fig. 2(a). In total, we have produced over 60 tips with a CNT extending from them. We have further demonstrated that tips from unsuccessful trials can be recycled by etching away the gold and reprocessing the same tip, increasing the overall yield without the expense of a new tip chip. In general, the length of attached tubes varied, but most were found to protrude between 100 and $500 \mathrm{~nm}$ from the tip surface. This is a range conducive to scanning over even fairly large features. The protrusion angles with respect to the cone axis of these useful tips were found to have an

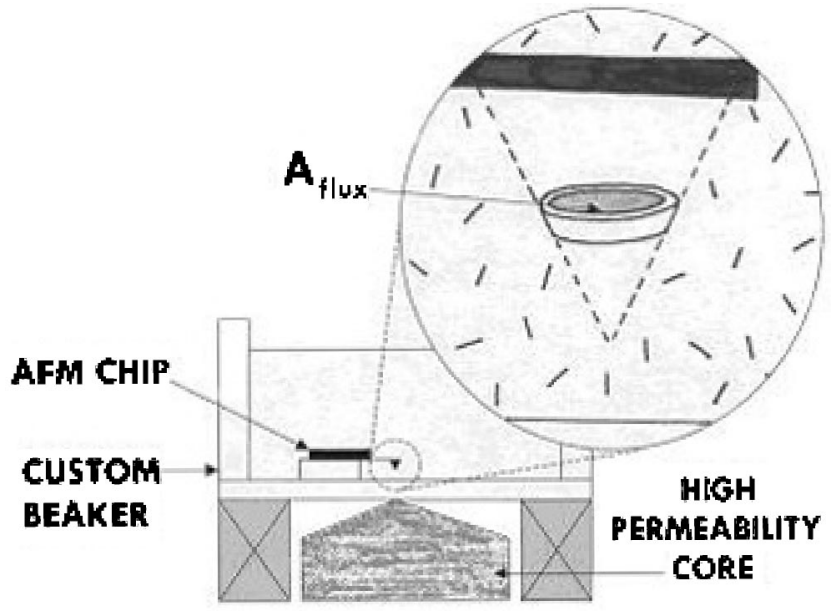

FIG. 1. Schematic of the experimental apparatus atop a solenoid. The inset shows the area through which the changing flux goes. 


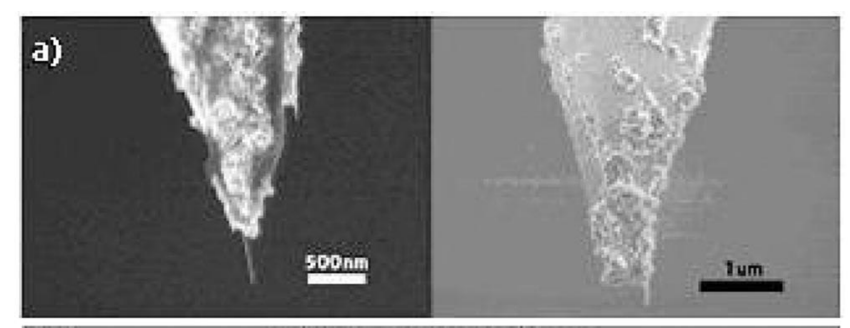

b)

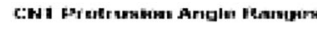

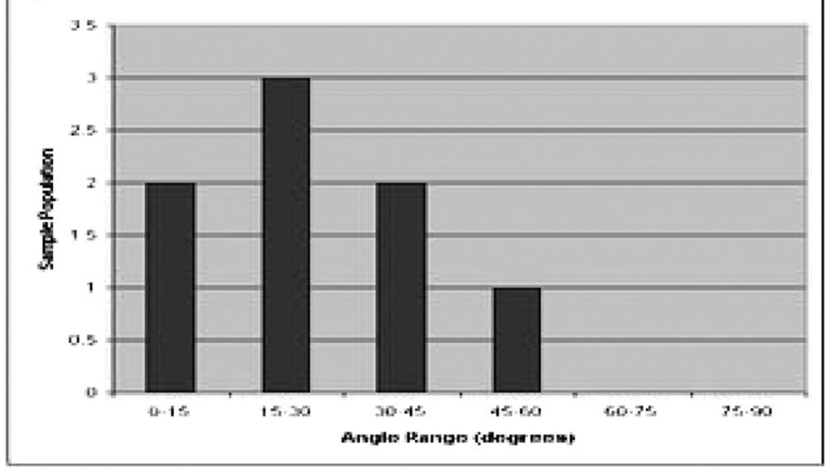

FIG. 2. (a) Examples of nanotube functionalized probes prepared with our method and (b) population densities of protrusion angle ranges for eight processed tips.

average value of about $35^{\circ}$, where $0^{\circ}$ is parallel with the tip axis and ideal for imaging. Population data of measured angle ranges for eight samples are shown in Fig. 2(b) and they are consistent with our expectations of an approximate $\cos \theta$ dependence of alignment with the field.

Control experiments involving simple introduction and removal of the tips into and from the suspension without energizing the magnetic coil were shown to attach some material to the probe surface. In these samples, however, the majority of the attached material was amorphous contamination. Few CNTs were found to lay flat against the surface rather than protruding from the end of the tip. No useful CNT-tipped probes were obtained in 20 control trials. Thus, the magnetic field appears to be necessary to obtain tips with a CNT extending from them. Similarly, the introduction of a direct current onto the solenoid failed to attract a CNT to the AFM tip and did not result in useful probes.

A plausible explanation for the effectiveness of the method may be the attraction between dipoles induced in the CNT and the metal film on the AFM. Superposing fields from slices of the conical tip, we find the highest concentration of force at the end of the AFM tip. Given that a change in flux is proportional to $A_{\text {flux }} B_{\mathrm{o}} \omega$ (Ref. 12) (see Fig. 1), we expect an induced current encircling the tip $I$ $\approx\left(A_{\text {flux }} A_{\text {current }} B_{\mathrm{O}} \omega\right) /(\rho L)$, where $\omega$ is the frequency, $\rho$ is the resistivity of the gold coating, $L$ is the average circumferential path length around the tip, $A_{\text {current }}$ is the total crosssectional area of the gold coating on the tip, and $A_{\text {flux }}$ is the area through which the magnetic flux threads. The induced dipole of the probe is on the order of $10^{-11} \mathrm{~A} \mathrm{~m}^{2}$. The average induced dipole on the multiwall CNT, is on the order of $10^{-23} \mathrm{~A} \mathrm{~m}^{2}$. This diamagnetic effect can be up to two orders of magnitude larger than the paramagnetic component expected from published susceptibility data, ${ }^{13,14}$ allowing the attractive force to be dominant. Modeling the gold coating of the AFM probe as parallel rings (Fig. 1, inset), we can evalu-
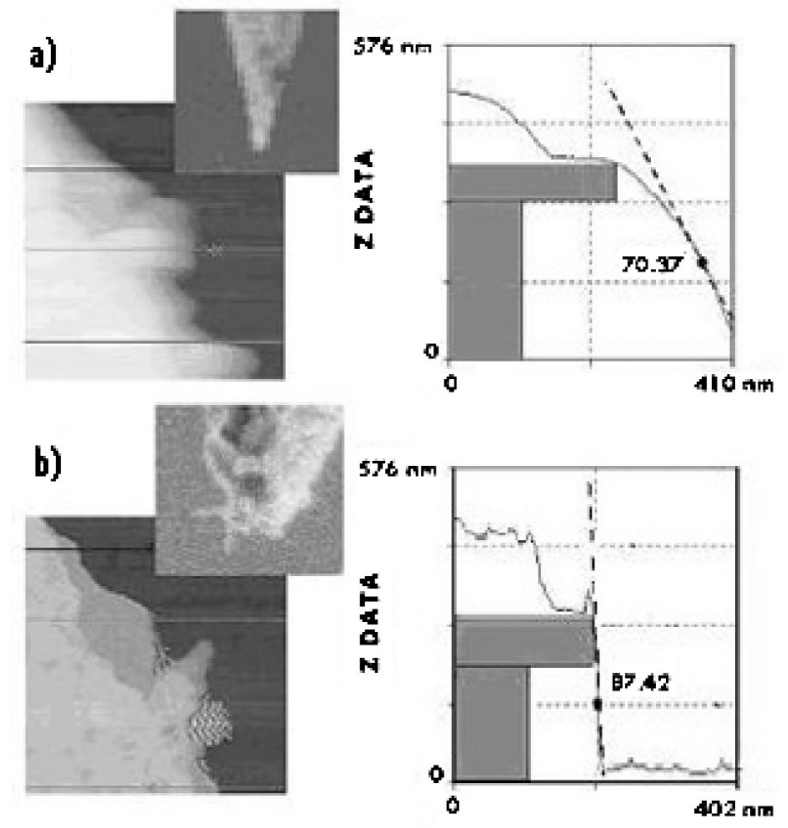

FIG. 3. AFM images and a typical graph of the cross section of an HFundercut metal surface taken by (a) a conventional tip and (b) a nanotube tip prepared with our method. Shown is the angle of the line with the horizontal taken at the indicated point.

ate the the potential energy between the two induced dipoles: ${ }^{12} U(r)=\left(-\mu_{\mathrm{o}} / 2 \pi r^{3}\right) m_{\text {tube }} m_{\text {probe }}$, where $r$ is the distance between the probe tip and the CNT, and $m_{\text {tube }}$ and $m_{\text {probe }}$ are the dipole moments of the tube and the probe, respectively. Assuming that the attraction must overcome translational thermal energy $\left[\sim(3 / 2) k_{B} T\right]$ of the CNT in the suspension, we find that the dipole attraction can dominate for separations on the order of $1 \mu \mathrm{m}$. Considering an estimated tube density in the suspension of $10^{4}$ tubes per $\mathrm{mm}^{3}$, we suppose that a CNT may be found occasionally within this distance due to convection and Brownian motion and, that in agreement with our results, we might form useful tips in a modest fraction of the time. Thus, it is plausible that the suspended CNTs are both aligned by the magnetic field and attracted to the tip by magnetophoresis. Upon reaching the tip surface, the CNT is probably held in place by van der Waals interactions after the oscillating field is removed.

In order to demonstrate the effectiveness of the resulting probes, we recorded atomic force microscopy images both with a CNT-modified tip and with a virgin probe of the same brand. The surface was an undercut metal lead on a silicon substrate. Thus, an ideal cross-sectional AFM image of the edge of the metal would show a $90^{\circ}$ angle. In the presented experiments, edge angle measurements were taken on the same area of the undercut sample before and after attachment of a CNT to the tip. The virgin probe yielded a $78^{\circ}$ edge at best, which is consistent with the listed probe spec of $80^{\circ}$. The CNT-modified tip achieved a consistent value of $87^{\circ}-88^{\circ}$. This shows a decrease in the profile error from $13^{\circ}-20^{\circ}$ to about $3^{\circ}$ or a relative increase in precision of about $80 \%$. We also noted that the mechanical response of the CNT-modified AFM tips differed from the virgin tips, as is apparent from feedback oscillations in the image in Fig. 3(b). We suppose this is due to our failure to optimize feedback parameters for the modified tips and to the inherent icense or copyright; see http://apl.aip.org/about/rights_and_permissions 
complexity of feedback control for two spring constants (cantilever and CNT bending) in series.

We have developed a simple, reliable, and inexpensive technique for the high yield assembly of a CNT onto AFM probes. The procedure involves only two steps: Metallization of scanning probes and placement of the probes in a nanotube suspension accompanied by an alternating magnetic field. It requires little time and no demanding control. It is possible that in addition to aligning the CNT, the oscillating magnetic field provides an attraction between the CNT and the probe tip. Images of chemically undercut metal structures (Fig. 3) provide evidence of the increased effectiveness of tips prepared using this method. The "cone angle" effect of the tip shape for the virgin AFM probe is about $70^{\circ}-77^{\circ}$. In contrast, the artifact for the CNT tips is $87^{\circ}-88^{\circ}$, or much closer to the ideal form factor of $90^{\circ}$. A concomitant increase in detail is apparent in the lateral resolution of the topographic images taken with the latter probes.

The authors would like to thank Dr. J. P. Lu for helpful discussions on magnetic interactions of CNTs and acknowl- edge the Office of Naval Research and the National Science Foundation for financial support of this work.

${ }^{1}$ M. R. Falvo, G. J. Clary, R. M. Taylor II, V. Chi, F. P. Brooks Jr., S. Washburn, and R. Superfine, Nature (London) 389, 582 (1997).

${ }^{2}$ E. W. Wong, P. E. Sheehan, and C. M. Lieber, Science 277, 1971 (1997).

${ }^{3}$ H. Dai, E. W. Wong, and C. M. Lieber, Science 272, 523 (1996).

${ }^{4}$ C. L. Cheung, J. H. Hafner, and C. M. Lieber, Proc. Natl. Acad. Sci. U.S.A. 97, 3809 (2000).

${ }^{5}$ H. J. Dai, J. H. Hafner, A. G. Rinzler, D. T. Colbert, and R. E. Smalley, Nature (London) 384, 147 (1996).

${ }^{6}$ S. Akita, H. Nishijima, Y. Nakayama, F. Tokumasu, and K. Takeyasu, J. Phys. D 32, 1044 (1999).

${ }^{7}$ H. Nishijima, S. Kamo, S. Akita, Y. Nakayama, K. I. Hohmura, S. H. Yoshimura, and K. Takeyasu, Appl. Phys. Lett. 74, 4061 (1999).

${ }^{8}$ E. W. Wong, P. E. Sheehan, and C. M. Lieber, Appl. Phys. Lett. 73, 3465 (1998).

${ }^{9}$ J. P. Lu, Phys. Rev. Lett. 74, 1123 (1995).

${ }^{10}$ B. W. Smith, Z. Benes, D. E. Luzzi, J. E. Fischer, D. A. Walters, M. J. Casavant, J. Schmidt, and R. E. Smalley, Appl. Phys. Lett. 77, 663 (2000).

${ }^{11}$ T. W. Ebbesen and P. M. Ajayan, Nature (London) 358, 16 (1992).

${ }^{12}$ D. J. Griffiths, Introduction to Electrodynamics, 3rd ed. (Prentice-Hall, Englewood Cliffs, NJ, 1999).

${ }^{13}$ A. P. Ramirez, R. C. Haddon, O. Zhou, R. M. Fleming, J. Zhang, S. M. McClure, and R. E. Smalley, Science 265, 84 (1994).

${ }^{14}$ F. Tsui, L. Jin, and O. Zhou, Appl. Phys. Lett. 76, 1452 (2000). 